\title{
Valve-sparing aortic root repair without down-sizing of the annulus
}

\author{
Paul P. Urbanski, MD, PhD, Xiaochun Zhan, MD, Husam Hijazi, MD, Michael Zacher, MD, and \\ Anno Diegeler, MD, $\mathrm{PhD}$
}

Objectives: The study was conducted to evaluate the intermediate results after patient-tailored aortic root repair with replacement of isolated sinuses adjusted to the existing aortic annulus diameter rather than annulus downsizing.

\begin{abstract}
Methods: Between 2000 and 2009, 236 patients with aortic root disease underwent patient-tailored restoration of the aortic root according to its anatomopathologic condition with repair of 1,2 , or all 3 sinuses in 73,94 , and 69 patients, respectively. Concomitant cusp repair was performed in 106 patients. Insufficiency grades of 0/1+, $2+, 3+$, and $4+$ were presented in 59, 74, 74, and 29 patients, respectively. All data were collected prospectively and intention-to-treat analysis was performed.
\end{abstract}

Results: Valve repair was successful in all but 6 patients. At the most recent clinical and echocardiographic follow-up of the remaining patients, which averaged $43 \pm 24$ months (range, 0.3-115 months), 216 patients showed no or slight aortic regurgitation (grade 0/1+) and 10 patients showed grade $2+$. Another 4 patients had grade $3+$ regurgitation and underwent aortic valve replacement. The actuarial freedom from aortic regurgitation grade $3+$ or more at 8 years was $95.2 \%$. Multivariate logistic regression analysis identified cusp repair as the independent risk factor for an aortic regurgitation grade of $2+$ or more with an odds ratio of $10.9(95 \%$ confidence interval, 2.5-47.7; $P=.002$ ). The association between aortic annulus size and functional results after repair could not be demonstrated.

Conclusions: Aortic root repair with replacement of isolated sinuses adjusted to the existing aortic annulus leads to excellent, durable results and can be recommended. Cusp disease is a leading aspect affecting functional results of repair. Therefore, establishment of reproducible cusp repair techniques is of utmost importance for further development of reconstructive aortic valve surgery. (J Thorac Cardiovasc Surg 2012;143:294-302)

Supplemental material is available online.

Even if the understanding of the aortic insufficiency mechanism in aortic root disease has improved since the introduction of the pioneering valve-sparing aortic root repair methods, ${ }^{1,2}$ the precise pathophysiology of aortic regurgitation is still a subject of controversy; consequentially, there is no agreement about an optimal surgical technique for root repair. It especially remains unclear whether downsizing of the annulus is generally indicated. The study was conducted to evaluate the intermediate results after patient-tailored aortic root repair with replacement of isolated sinuses adjusted to the existing annulus diameter.

From Cardiovascular Clinic, Bad Neustadt, Germany.

Disclosures: Dr Urbanski discloses a financial relationship with MAQUET Cardiovascular, Inc.

Read at the 91st Annual Meeting of The American Association for Thoracic Surgery, Philadelphia, Pennsylvania, May 7-11, 2011.

Received for publication May 3, 2011; revisions received Sept 22, 2011; accepted for publication Oct 21, 2011; available ahead of print Nov 21, 2011

Address for reprints: Paul P. Urbanski, MD, PhD, Herz- und Gefaess-Klinik, Salzburger Leite 1, 97616 Bad Neustadt, Germany (E-mail: p.urbanski@ herzchirurgie.de). $0022-5223 / \$ 36.00$

Copyright (c) 2012 by The American Association for Thoracic Surgery doi:10.1016/j.jtcvs.2011.10.056

\section{PATIENTS AND METHODS}

Between September 2000 and January 2011, a total of 322 patients underwent aortic root repair at our facility. In all but 15 patients, in whom the reimplantation technique was used during surgical workshops for demonstrations, the patient-tailored aortic root remodeling with replacement of isolated sinuses, described by us originally as the single-patch technique, was used. ${ }^{3-5}$ For the purpose of this study, 236 consecutive patients operated on using this technique until September 2009 were enrolled into assessment to achieve a follow-up time of at least 1 year.

All patients had aortic root disease such as chronic aneurysm $(n=206)$ or acute aortic dissection $(n=30)$. Nine patients had Marfan disease and 37 had bicuspid aortic valves. Insufficiency grades of $0 / 1+, 2+, 3+$, and $4+$ were present in 59, 74, 74, and 29 patients, respectively. The etiology was degenerative in 146, atherosclerotic in 8, and mixed in 71 cases. Four patients had had an inflammatory etiology. The detailed patient and operative data are given in Table 1. At least once a year, the patients were followed up by echocardiography performed in our outpatient clinic or by referring cardiologists.

\section{Surgical Technique}

Aortic root repair using the original single-patch technique has been described previously. ${ }^{3-5}$ In short, to choose the proper aortic graft size, we measured the aortic annulus with a valve sizer and defined it as the same size as the biggest sizer that could pass through the aortic valve. Because it is not possible to pass the sizer in bicuspid valves or in pronounced septal hypertrophy, the sizer was only placed on the valve, and the appropriate size was judged visually. The tube with the same diameter as the aortic annulus or $1 \mathrm{~mm}$ bigger was chosen. The distorted sinuses of Valsalva were excised, leaving a 2- to 3-mm rim of aortic wall attached to the aortic valve. Depending on the number of sinuses that had to be 
replaced, 1 to 3 patches were excised from the vascular graft and trimmed to teardrop shapes matching the size of the respective valve cusps, keeping in mind that the sum of the sinuses' widths had to be equal to the circumference of the tube graft chosen. Until 2006, we used a standard straight woven polyester graft (InterGard) for the root repair, and thereafter, a special graft with an uncrimped proximal part (InterGard Woven Aortic Thoracic Graft; InterVascular, MAQUET Cardiovascular, La Ciotat, France) because cutting the patches from the uncrimped tube facilitates the determination of their proper size and shape and improves the tightness of the suture line by eliminating leaks between the folds of Dacron fabric. ${ }^{5}$ The patches were sewn to the rim of the aortic wall with a 5-0 polypropylene running suture, and coronary buttons were reimplanted in cases in which the coronary sinuses were replaced. No tissue glue was used, either in the repair of chronic aneurysms or in acute dissections.

Several additional procedures on the cusps, such as free margin plication, reinforcement, cusp patch plasty, or others, completed the aortic root repair, if necessary. Warfarin was not given postoperatively except in patients with atrial fibrillation or other specific indications.

\section{Statistical Analysis}

All perioperative data were collected prospectively and an intention-totreat analysis was performed. The end point of follow-up was death or aortic valve replacement from any cause. Categorical variables are reported as frequencies and continuous variables are reported as mean \pm standard deviation unless otherwise indicated. The Kaplan-Meier method was used to estimate the long-term survival or freedom from valve-related events and the multivariable Cox regression model was built to determine the independent multivariable predictors of late outcomes. Statistical analysis was performed with the SPSS software version 18.0 (SPSS Inc, Chicago, Ill).

\section{RESULTS}

The mean crossclamp time, including the period of circulatory arrest and cerebral perfusion, was $107 \pm 30$ minutes and ranged from 45 minutes for a simple case to $193 \mathrm{~min}$ utes of cumulative time for a complex aortic root and valve repair and rerepair during a second run; this occurred in 3 patients. Aortic root repair consisted of 1, 2, or all 3 sinuses of Valsalva in 73, 94, and 69 patients, respectively. When 1 sinus was repaired, it was most frequently the noncoronary $(\mathrm{n}=71)$ and, as an exception, the right coronary $(\mathrm{n}=2)$. In patients with repair of 2 sinuses, it was a combination of the right and noncoronary sinuses in 93 patients and right and left coronary sinuses in only 1 patient. Concomitant cusp repair was performed in 106 patients, among whom repair of all 3 sinuses of Valsalva was necessary in 25 patients. The cusp repair consisted of plicating the central cusp portion ( 23 cusps), a technique that has been used throughout the study duration and up to the present. The techniques of free margin reinforcement (12 cusps) and commissural plication ( 15 cusps) had to be completed occasionally within a corresponding subcommisural trigone by subcommisural annuloplasty to improve the coaptation of the restricted cusps rather than to downsize the entire annulus. These were performed until 2008, after which they were replaced by cusp patch plasty (68 cusps) with autologous pericardium. ${ }^{6,7}$

Operative data and concomitant procedures are shown in Table 2.
TABLE 1. Patient characteristics

\begin{tabular}{lc}
\hline & No. (\%) or mean \pm SD \\
\hline No. of patients & $236(100)$ \\
Age (y) & $63 \pm 13$ \\
Male & $156(66.1)$ \\
Sinus rhythm & $214(90.7)$ \\
Functional class NYHA $\geq$ III & $90(38.1)$ \\
Aortic insufficiency $\geq 2+$ & $177(75.0)$ \\
Bicuspid aortic valve & $37(15.8)$ \\
Marfan & $9(3.8)$ \\
Acute aortic dissection & $30(12.7)$ \\
Ejection fraction $\leq 35 \%$ & $11(4.7)$ \\
Concomitant diseases & \\
$\quad$ Hypertension & $193(81.8)$ \\
Coronary heart disease* & $46(19.5)$ \\
COPD & $24(10.2)$ \\
Previous neurologic events & $19(8.1)$ \\
$\quad$ With residuals & $8(3.4)$ \\
$\quad$ Without residuals & $11(4.7)$ \\
Renal failure & $23(9.8)$ \\
With hemodialysis & $1(0.4)$ \\
Previous cardiac surgery & $8(3)$ \\
$S D$, Standard deviation; $N Y H A$, New York Heart Association class; COPD, chronic
\end{tabular}

$S D$, Standard deviation; $N Y H A$, New York Heart Association clas;
obstructive pulmonary disease. *With surgical relevant stenosis

\section{Operative Mortality and Morbidity}

There were 3 deaths during the 30 days after surgery. The first patient (female, 56 years old) had undergone an ascending aortic replacement in 1996 because of acute aortic dissection. During that operation, French glue was used to approximate the dissected layers in the region of the aortic root as well as in the aortic arch. A large false aneurysm later developed at the distal suture line and was treated successfully with a complete arch replacement in 1999. Four years later, she had an aneurysm at the proximal suture line combined with mild aortic insufficiency. The early postoperative course after aortic root repair with

\section{TABLE 2. Operative data}

\begin{tabular}{lc}
\hline & No. $(\%)$ or mean \pm SD \\
\hline CPB time (min) & $160 \pm 43$ \\
Aortic clamp time (min) & $107 \pm 30$ \\
Concomitant procedures & \\
Partial arch replacement & $101(42.8)$ \\
Total arch replacement & $61(25.8)$ \\
CABG & $46(19.5)$ \\
Myectomy & $8(3.4)$ \\
Mitral valve repair & $6(2.5)$ \\
ASD closure & $6(2.5)$ \\
Tricuspidal valve repair & $1(0.4)$ \\
Aortic aneurysm diameter $(\mathrm{mm})$ & $55.4 \pm 9.2$ \\
Aortic annulus diameter $(\mathrm{mm})$ & $26.1 \pm 2.8$ \\
Vascular graft diameter $(\mathrm{mm})$ & $26.9 \pm 3.1$ \\
STJ diameter after surgery $(\mathrm{mm})$ & $26.7 \pm 3.1$ \\
\hline
\end{tabular}

$S D$, Standard deviation; $C P B$, cardiopulmonary bypass; $C A B P$, coronary artery bypass grafting; $A S D$, atrial septal defect; $S T J$, sinotubular junction. 

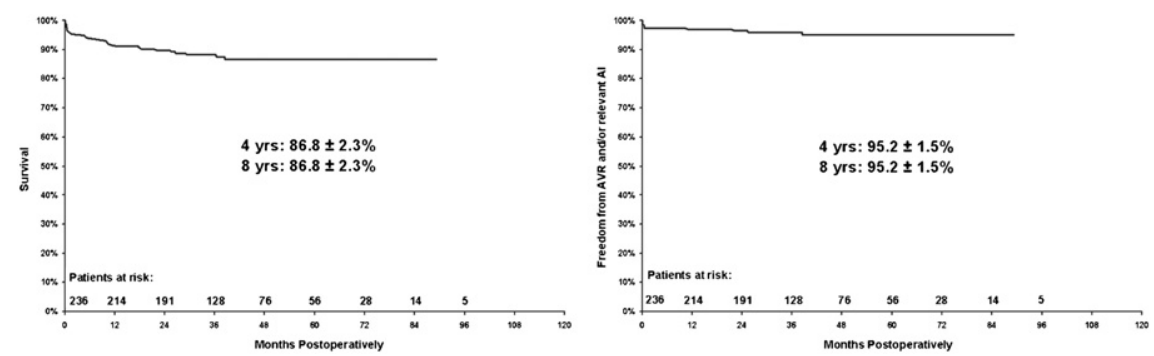

FIGURE 1. Left, Actuarial survival without aortic valve replacement. Right, Freedom from relevant ( $\geq 3+$ ) aortic insufficiency (AI) and/or aortic valve replacement $(A V R)$. Note that in the series presented all patients with relevant AI underwent AVR.

replacement of all 3 sinuses was uneventful, apart from a rethoracotomy for bleeding and atrial fibrillation treated with sotalol. She died suddenly on the 15 th postoperative day. At autopsy, no morphologic disease was discovered; thus a rhythm disorder was presumed to be the cause of death. Another patient (male, 79 years old) with chronic atherosclerotic aneurysm and aortic valve insufficiency died suddenly on the seventh day after aortic root and complete arch repair owing to malignant arrhythmia resulting in cardiac arrest after an otherwise uneventful postoperative course. Also in this case, no pathologic conditions were found at autopsy apart from severe left ventricular hypertrophy. The third patient (female, 76 years old) with acute aortic dissection had a heparin-induced thrombocytopenia after surgery and died on the 26th day of multiple organ failure.

The in-hospital mortality was $2.1 \%$ because there were 2 further deaths that occurred in-hospital but after the 30-day time range. In 1 patient with chronic aortic aneurysm and severe aortic insufficiency combined with coronary heart disease and poor ventricular pump function, ventricular fibrillation occurred on the fourth postoperative day and neurologic injury developed after cardiopulmonary resuscitation. He died of subsequent pneumonia. Another patient with a history of nephrectomy had renal failure, necessitating dialysis, and respiratory insufficiency, necessitating prolonged ventilation. Pneumonia and subsequent sepsis developed, and he died of multiple organ failure.

Sixteen patients had to be reintubated owing to respiratory insufficiency, the most frequent postoperative complication. Twelve patients required tracheotomy. Temporary dialysis was necessary in 5 patients.

Permanent neurologic deficit or temporary dysfunction occurred in $3(1.3 \%)$ and $7(3.0 \%)$ patients, respectively.

Rethoracotomy for bleeding was necessary in $13(6 \%)$ patients in the entire population and in $7(23 \%)$ patients of the acute dissection subgroup. No blood transfusion was required in $108(45.8 \%)$ patients.

\section{Operative Functional Results}

The aortic root and valve repair was functionally successful in all but 6 patients; there were 2 conversions and 4 valve replacements after echocardiographic control.
The postoperative echocardiography performed during the hospital stay showed no or trivial insufficiency in 225 patients and mild-to-moderate (2+) insufficiency in 5 patients. The mean gradient across the valve was $4.9 \pm 2.7$ $\mathrm{mm} \mathrm{Hg}$.

\section{Late Mortality and Morbidity}

Follow-up was completed for all but 2 patients who could no longer be located after 1 and 2 years, respectively. It averaged $43 \pm 24$ months (range, 0-115 months) for all patients, totaling 846 patient-years. There were 15 late deaths: 10 noncardiac, 4 cardiac (congestive heart failure, 2; myocardial infarction, 1; and mitral valve endocarditis, 1) and 1 sudden death. Thus, the overall patient survival, including early mortality, was $91.9 \%$ at the last follow-up; however, no death was related to the aortic valve, and the survival of patients without aortic dissection and younger than 60 years was $98.5 \%$. The actuarial survival without aortic valve replacement is illustrated in Figure 1.

After discharge, there were 4 further aortic valve replacements during the follow-up (see also late functional results). A relevant aortic valve insufficiency occurred in all these cases; however, in 1 case, a vascular graft infection led to aortic valve endocarditis and subsequent insufficiency. The patient underwent complete root replacement with a valve composite graft and survived, as did all 9 remaining study patients in whom aortic valve replacement was performed.

There were 3 embolic events. All of them were cerebral and occurred only among the 21 patients having permanent or paroxysmal atrial fibrillation. A total of 26 patients were given warfarin, mostly because of atrial fibrillation. Spontaneous bleeding occurred in 2 of them, with a lethal outcome in 1 of them.

\section{Late Functional Results}

Among the 230 patients with a successfully repaired aortic valve, 216 patients showed no or slight aortic regurgitation (grade 0/1+) and 10 patients showed grade 2+. Four patients had grade $3+$ regurgitation and underwent an aortic valve replacement, on average 24 months (range, 11-39 months) after repair. All 10 patients who finally underwent 


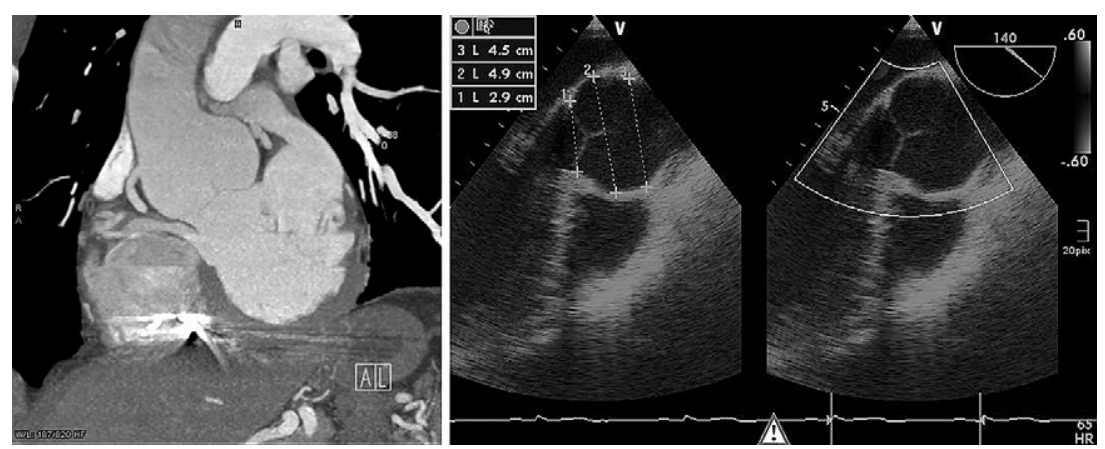

FIGURE 2. Computed tomographic angiogram and echocardiogram of a 32-year-old patient with Marfan disease without aortic regurgitation showing physiologic proportions between the annulus $(29 \mathrm{~mm})$ and cusp sizes despite root enlargement.

aortic valve replacement had aortic cusp repair in common, which consisted of commissural plication, free margin reinforcement, and central plication in 7,2, and 1 case, respectively. None of them had cusp patch plasty with autologous pericardium. Multivariate logistic regression analysis identified cusp repair as the independent risk factor for aortic regurgitation grade $2+$ or more, with an odds ratio of 10.9 (95\% confidence interval, 2.5-47.7; $P=.002$ ). An association between aortic annulus size and functional results after repair could not be demonstrated. No change of aortic annulus size and/or change of aortic root size and form were revealed in any patient. The actuarial freedom from aortic regurgitation grade $3+$ or more and/or aortic valve replacement owing to any cause at 8 years was $95.2 \%$ (Figure 1).

\section{DISCUSSION}

From a functional point of view, the aortic valve should be considered as a unit consisting of the aortic root and
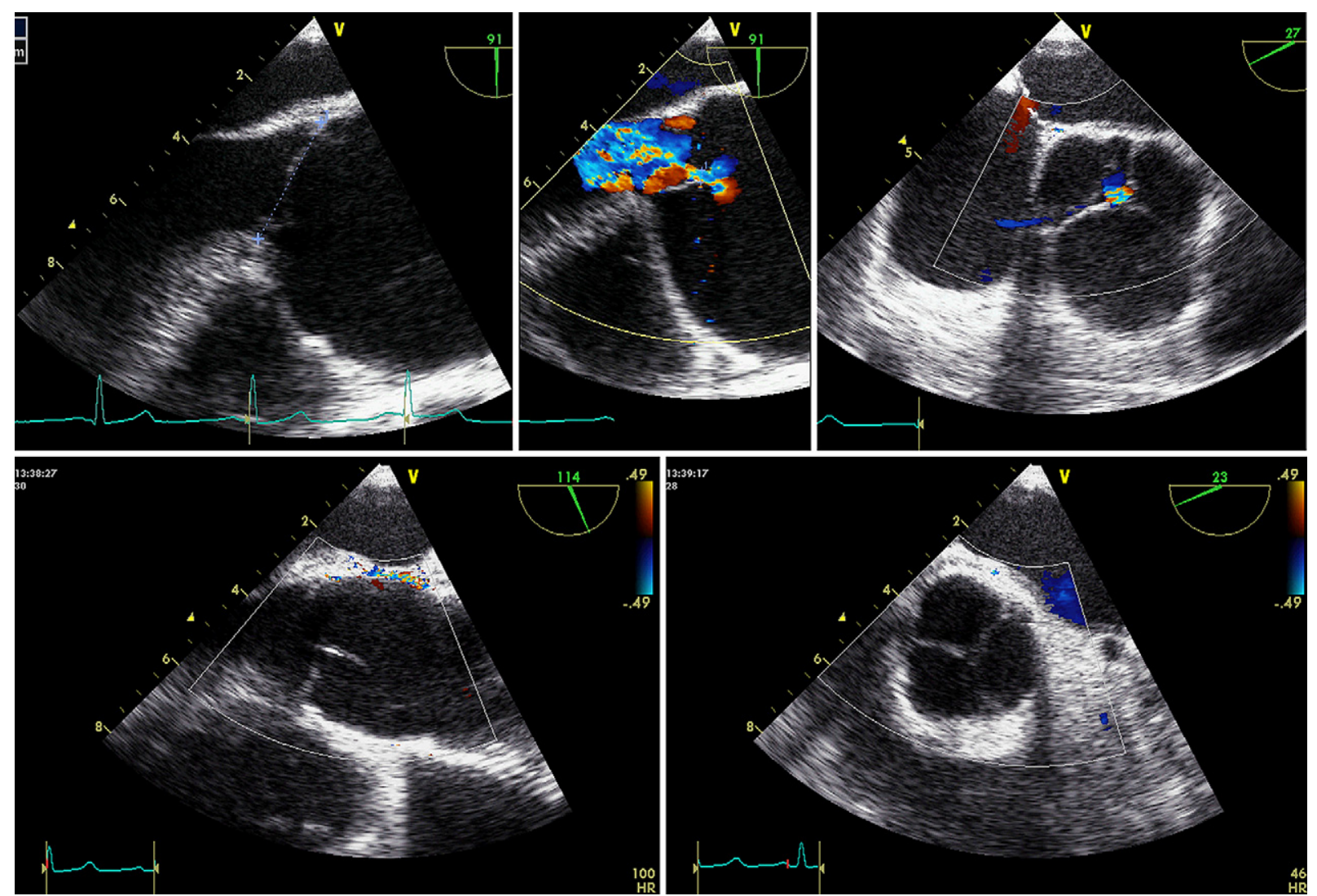

FIGURE 3. Preoperative (upper row) and postoperative (bottom row) transesophageal echocardiograms showing central aortic regurgitation in a 38-yearold patient with pronounced dilatation of the right and noncoronary sinuses and an annulus size of $30 \mathrm{~mm}$. The regurgitation was completely abolished after isolated repair of dilated sinuses using the single-patch technique. 

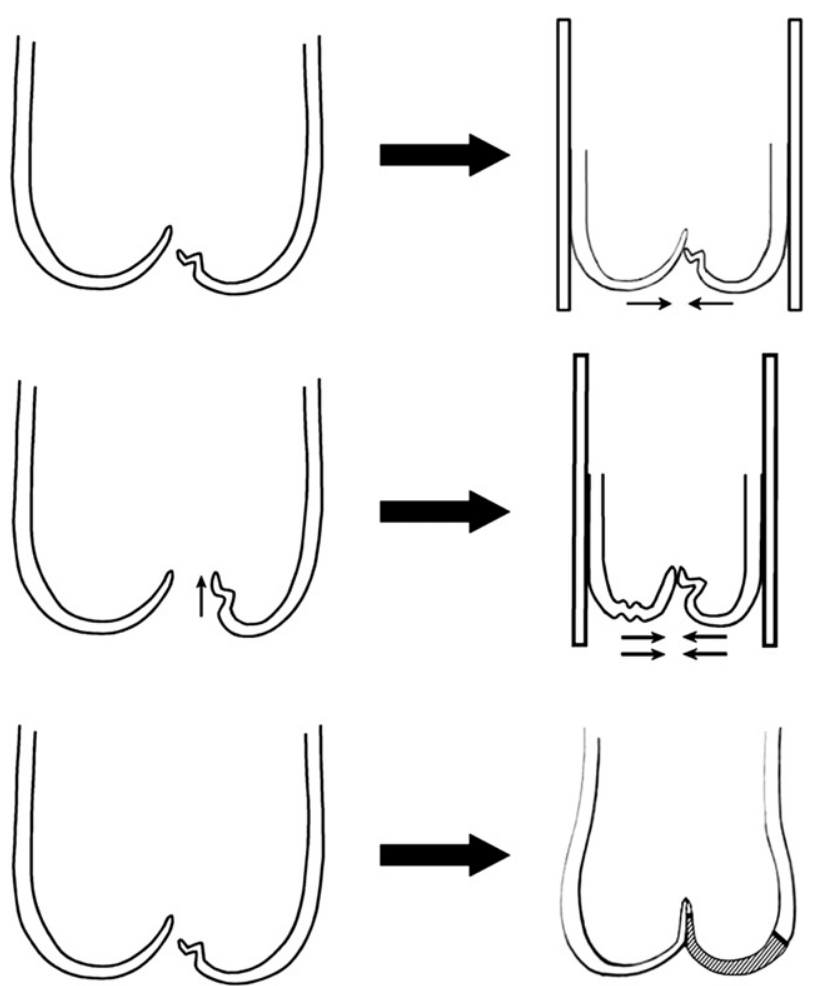

FIGURE 4. Schematic illustration showing mechanisms of aortic root and valve repair. Upper row, Downsizing (arrows) the complete aortic root (including annulus) improves cusp coaptation but does not normalize it. Middle row, By shortening the free margin, cusp height can be increased (single arrow), if necessary, but its effective surface becomes more restricted, necessitating drastic downsizing (double arrows). This may lead to folding of unchanged cusps and hindering of valve motion. Bottom row, Remodeling the aortic root and restoring cusp size with pericardium (see also Figure 6).

cusps; hence, the clinically relevant aortic regurgitation can be caused by isolated changes of the valve cusps (type I), isolated changes of the sinuses of Valsalva (type II), or a combination of both (type III). Because of the variety of aortic root and valve diseases, we propose this simple classification, which, despite its simplicity, indicates the necessary surgical approach. The pathomechanism of aortic insufficiency can be explained by any abnormality of the anatomophysiologic balance between the size and shape of the sinuses of Valsalva and the size and shape of the aortic cusps, which almost always correspond to the diameter of the aortic annulus. ${ }^{8-10}$ This relationship can even be maintained when the absolute sizes of the aortic root and aortic annulus considerably exceed those measurements that are regarded as normal (Figure 2). On the other hand, most patients with aortic insufficiency have only slight dilatation of the aortic root with a diameter of about $4 \mathrm{~cm}$. As demonstrated in this study, the extent of the dilatation is not equal in all sinuses and most often spares the left coronary sinus (Figure 3). This phenomenon is also observed in acute aortic dissection in which the left coronary sinus is frequently not involved in aortic wall dissection. Even during aging, the sinuses alter irregularly, changing the root proportions only slightly. ${ }^{11}$ However, the same can be said about dilatation of the degenerative aortic wall owing to the nonuniform distribution of medial degeneration within the ascending aorta. ${ }^{12}$ Thus, if aortic insufficiency is caused by dilatation of an isolated sinus or sinuses and there is no additional cusp disease to be addressed surgically, the regurgitation can, as demonstrated in our study, be abolished by repair of the particular sinuses alone, leading to excellent and lasting function, regardless of the aortic annulus diameter (Figure 3).

However, during the dilatation process, the sinuses not only alter irregularly, they change their dimensions in the transverse and longitudinal axes, leading to distal movement of the commissures (especially between the noncoronary and the right coronary sinuses, which is most often the highest one) and tension of the cusp free margin. This results in the development of a prolapse and commissural cusp fenestrations and secondary shrinking or folding of the cusp body with subsequent restriction. ${ }^{7}$ In such cases, the dysfunctional cusp coaptation can be improved by downsizing the complete aortic root (eg, by reimplantation of the valve within a vascular tube) but it cannot be normalized (Figure 4). Hence, in patients with a pronounced restriction of an aortic cusp or cusps, more extensive downsizing is necessary, ${ }^{13,14}$ which can result in folding of the unchanged cusps (Figure 4). This can hinder the smooth cusp motion, leading even to the development of a functional stenosis, and can accelerate late cusp degeneration. Extensive downsizing is especially necessary in cases in which a plication of the free margin is made to increase the cusp height because it leads to a worsening of the restriction (Figure 5). Nevertheless, if the cusp coaptation dysfunction is not very pronounced, some downsizing of the valve can be helpful to abolish, or at least to decrease, aortic regurgitation. This, besides excellent hemostatic characteristics of the proximal anastomosis, speaks to the advantages of the reimplantation technique. At the same time, the necessity of repairing all sinuses, even if not all are diseased, and the choice of the appropriate tube size remain a challenge. On the other hand, in experimental settings it has been demonstrated that aortic root remodeling provides smoother valve movement than reimplantation. ${ }^{15-17}$ The experimental settings, in which normal aortic valves are examined, especially those without any cusp disease, can be compared with the clinical experience showing that the valve function after aortic root remodeling remains long lasting if the valve cusps are unchanged and the primary result of surgery is satisfactory. ${ }^{18,19}$

However, we believe that our technique with patienttailored repair of isolated sinuses offers several advantages to the original aortic root remodeling. It allows any correction of the size and shape of the neosinuses, which is not 

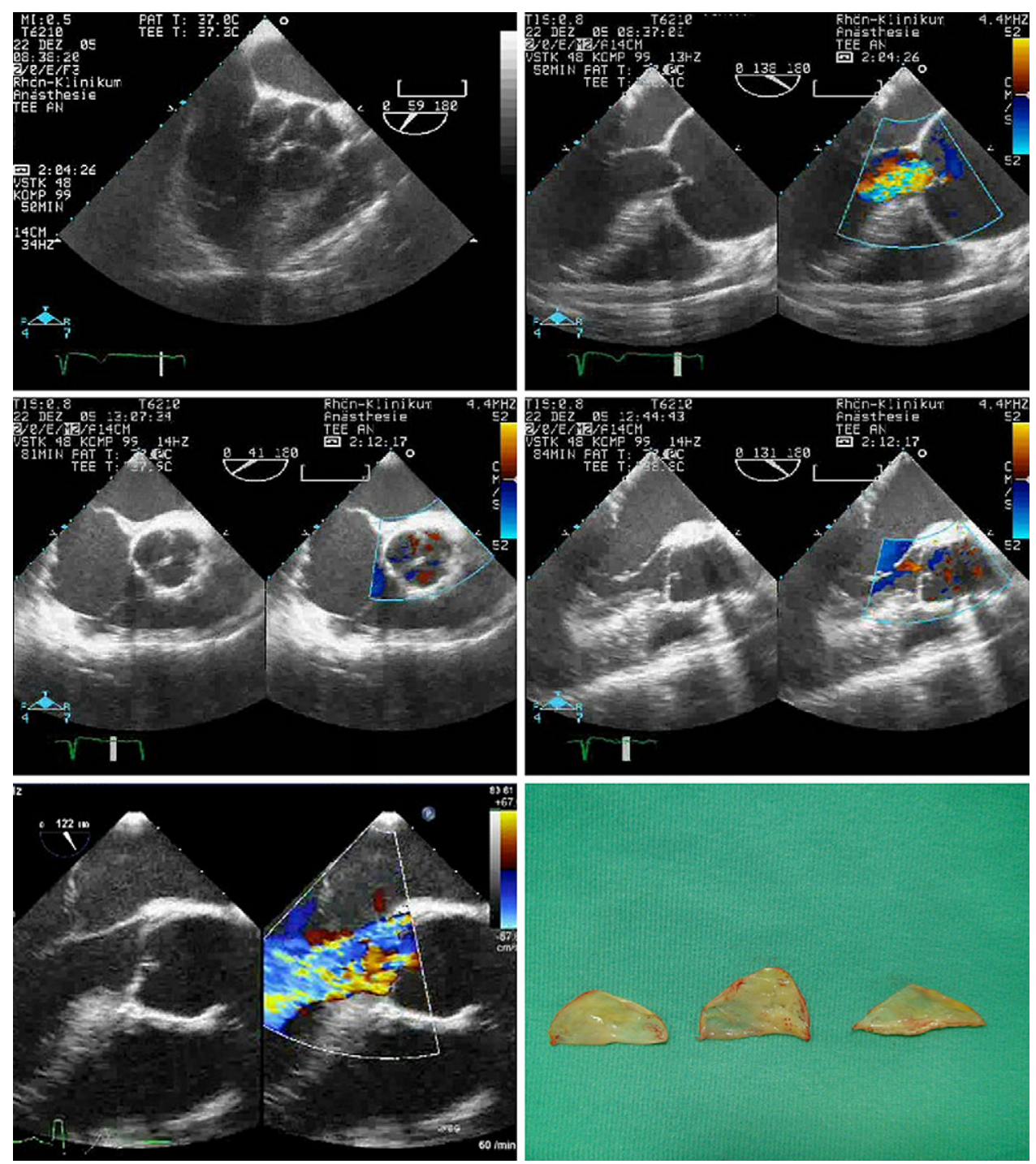

FIGURE 5. Upper row, Preoperative transesophageal echocardiogram of a 63-year-old patient with dilatation of the right and noncoronary sinuses and aortic regurgitation caused by prolapse and secondary restriction of the left coronary cusp. Aortic annulus: $29 \mathrm{~mm}$. Middle row, Postoperative transesophageal echocardiogram after repair of right and noncoronary sinuses, reinforcement of left coronary cusp free margin with continuous polytetrafluoroethylene suture, and subcommissural annuloplasty. Bottom row, left, Transesophageal control echocardiogram after 10 months showing recurrent regurgitation caused by progressive restriction of the left coronary cusp without any changes of aortic root size and shape. Bottom row, right, Aortic valve cusps excised during redo surgery showing progressive restriction of left coronary cusp.

possible when a tube is used and trimmed to the final crownshaped form. Additionally, it enables very precise sewing, passing the stitches exactly through the annulus rather than the aortic wall remnants, a characteristic that, in our opinion, is decisive for hemostatic tightness and lasting durability. Furthermore, it enables an individual case-based matching of the neosinuses to the corresponding cusps, and it ensures the creation of a lasting neosinotubular junction within the suture line between the repaired root and the tube. Last, it is advantageous when additional cusp repair is necessary because it facilities the reconstruction of the cusps that are not located deep inside the tube. Hence, after repair of isolated sinuses in patients without additional cusp disease, regardless of the number of sinuses repaired, neither was a recurrent aortic insufficiency observed nor did an adverse change of the aortic root size and/or form occur.

On the other hand, in patients with additional cusp disease, remaining or recurrent insufficiency occurred only in those patients in whom a cusp repair using techniques aimed at increasing the cusp height were performed (Figure 5). For this reason, we changed our approach to the cusp disease during our anatomic root remodeling.

Because we recognized cusp restriction as the leading pathophysiologic mechanism of aortic insufficiency, we at first introduced the technique of basal cusp enlargement ${ }^{6}$ and later established cusp body repair with autologous 

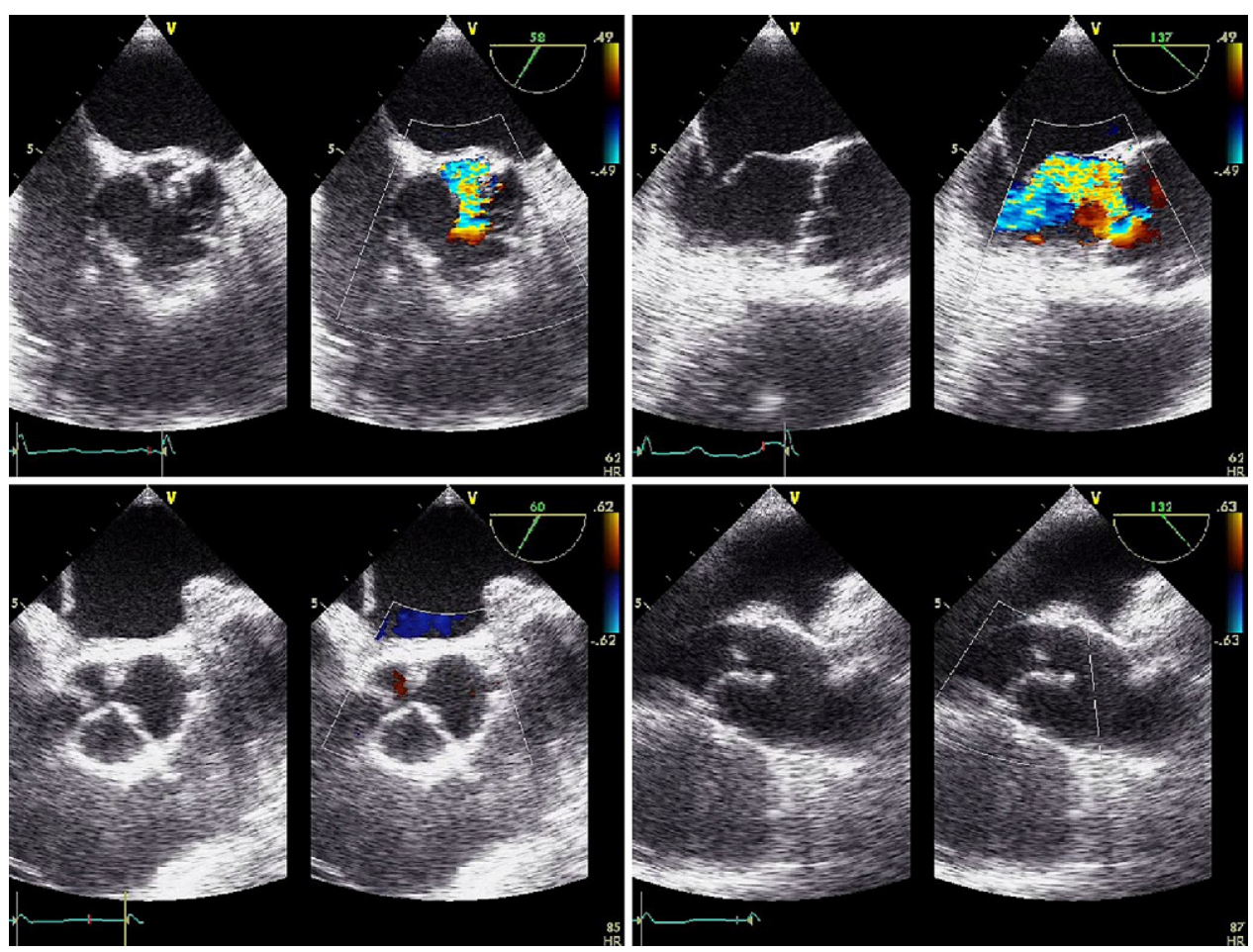

FIGURE 6. Upper row, Preoperative transesophageal echocardiograms of a 58-year-old patient with dilatation of the right and noncoronary sinuses and severe aortic insufficiency caused by prolapse and secondary restriction of the right coronary cusp. Aortic annulus: 30 mm. Bottom row, Postoperative transesophageal echocardiograms after repair of the right coronary cusp using autologous pericardium and isolated repair of dilated sinuses using the single-patch technique.

pericardium (Figure 6), ${ }^{7}$ Thereafter, we were able to increase the number of concomitant aortic root and valve repairs to more than 50 a year (Figure E1). More important, we did not observe remaining or recurrent relevant aortic regurgitation in any patient operated on in the series presented, nor in our entire experience up to date (101 further patients were operated on using single-patch technique between October 2009 and March 2011). If this technique had not improved functional results, one would have expected to observe an occurrence of remaining or recurrent aortic regurgitation in the recent patient cohort because the median time for emergence of relevant aortic insufficiency was 0.3 months in our series.

Nevertheless, among those study patients without additional cusp disease (type II), we have not observed remaining or recurrent aortic regurgitation during the entire follow-up period in any case, regardless of the aortic annulus diameter or the number of sinuses repaired. Regarding this point, the results reported recently by David, Maganti, and Armstrong, ${ }^{19}$ which revealed the patient's age as the only predictor of recurrent aortic insufficiency in either the remodeling or the reimplantation group, do not really diverge from ours. They even emphasize, in our opinion, the role of the cusps on long-term outcome because the cusps are the main subject of alteration during the process of valve aging.
In conclusion, isolated sinus repair can facilitate aortic root surgery considerably; however, the decision-making process for sparing some sinuses should include not only their size but also the disease etiology, the anatomopathology of the aortic root and valve, and the patient's age. If there are no cusp diseases to be repaired, aortic root reconstruction without downsizing of the annulus leads to excellent and durable results and can be recommended. A successful aortic root repair should target the restoration of the anatomophysiologic relationship between the root and the cusps, with the aortic annulus being the anatomic standard that can be used as a reference for this restoration. On the other hand, cusp disease is a leading aspect affecting functional results of repair. Therefore, we believe that establishment of reproducible cusp repair techniques is of utmost importance for further development of reconstructive aortic valve surgery.

We thank Ms Melissa Lindner, Ms Alexandra Metz, and Ms Bianca Müller for assistance in preparing this article.

\section{References}

1. David TE, Feindel CM. An aortic valve-sparing operation for patients with aortic incompetence and aneurysm of the ascending aorta. J Thorac Cardiovasc Surg. 1992;103:617-22.

2. Sarsam MAI, Yacoub M. Remodeling of the aortic valve annulus. $J$ Thorac Cardiovasc Surg. 1993;105:435-8. 
3. Urbanski PP. Valve-preserving aortic root reconstruction [Letter]. J Thorac Cardiovasc Surg. 2001;121:1220.

4. Urbanski PP. Valve-sparing aortic root repair with patch technique. Ann Thorac Surg. 2005;80:839-44

5. Urbanski PP, Zhan X, Frank S, Diegeler A. Aortic root reconstruction using new vascular graft. Interact Cardiovasc Thorac Surg. 2009;8:187-90.

6. Urbanski P. Basal cusp enlargement for repair of aortic valve insufficiency. J Thorac Cardiovasc Surg. 2010;139:98-102.

7. Urbanski P. Aortic valve insufficiency with extensive cusp pathology: repair with a pericardial patch. Eur J of Cardiothorac Surg. 2010;37:962-4.

8. Tubrikar MJ, Labrosse MR, Zehr KJ, Robicsek F. Aortic root dilatation may alter the dimensions of the valve leaflets. Eur J Cardiothorac Surg. 2005;28: 850-6.

9. Labrosse MR, Beller CJ, Robicsek F, Thubrikar MJ. Geometric modeling of functional trileaflet aortic valves: development and clinical applications. J Biomech. 2006;39:2665-72.

10. Swanson WM, Clark RE. Dimensions and geometric relationships of the human aortic valve as a function of pressure. Circ Res. 1974;22:871-82.

11. Vasan RS, Larson MG, Levy D. Determinants of echocardiographic aortic root size. The Framingham Heart Study. Circulation. 1995;91:734-40.

12. Aggozino L, Ferraraccio F, Esposito S, Trocciola A, Parente A, Della Corte A, et al. Medial degeneration does not involve uniformly the whole ascending aorta: morphological, biochemical and clinical correlations. Eur J Cardiothorac Surg. 2002;21:675-82.

13. Svensson LG, Cooper M, Batizy LH, Nowicki ER. Simplified David reimplantation with reduction of anular size and creation of artificial sinuses. Ann Thorac Surg. 2010;89:1443-7.

14. Kouchoukos NT, Milewicz DM, Elefteriades J, Svenson LG, David TE, El Khoury G, et al. Aortic surgery symposium 2010 discussions-session I: aortic root I and session II: aortic root II. J Thorac Cardiovasc Surg. 2010; 140(suppl):S45-51.

15. Leyh RG, Schmidtke C, Sievers HH, Yacoub MH. Opening and closing characteristic of aortic valve after different types of valve-preserving surgery. Circulation. 1999;100:2153-60.

16. Katayama S, Umetani N, Sugiura S, Hisada T. The sinus of Valsalva relieves abnormal stress on aortic valve leaflets by facilitating smooth closure. $J$ Thorac Cardiovasc Surg. 2008;136:1528-35.

17. Fries R, Graeter T, Aicher D, Reul H, Schmitz Ch, Böhm M, et al. In vitro comparison of aortic valve movement after valve-preserving aortic replacement. J Thorac Cardiovasc Surg. 2006;132:32-7.

18. Yacoub MH, Gehle P, Chandrasekaran V, Birks EJ, Child A, Radley-Smith R. Late results of a valve-preserving operation in patients with aneurysms of the ascending aorta and root. J Thorac Cardiovasc Surg. 1998;115:1080-90.

19. David TE, Maganti M, Armstrong S. Aortic root aneurysm: principles of repair and long-term follow-up. J Thorac Cardiovasc Surg. 2010;140:14-9.

\section{Discussion}

Dr Tirone E. David (Toronto, Ontario, Canada). I have no conflicts to disclose. I commend you, Paul, for these excellent clinical outcomes in patients with a variety of proximal aortic aneurysms with and without aortic insufficiency. Contrary to what your second slide showed, isolated aneurysms of the aortic sinuses do not cause aortic insufficiency. Aortic insufficiency is obviously caused by cusp disease, annular dilatation, and/or sinotubular junction dilation. Isolated sinus of Valsalva aneurysm causes no aortic valve dysfunction.

I believe your method to treat patients with dilated aortic sinuses is reasonable, but I am not sure it can be applied to all patients with proximal aortic root disease. The fact that your patients were 63 years old and only $38 \%$ had degenerative aneurysms is an indication that you have included a variety of diseases that cause aortic insufficiency and/or aortic sinus aneurysm. Your patient population is composed of a mix of aneurysmal diseases that in my view primarily affected the ascending aorta, and the sinuses became secondarily involved.
Reimplantation of the aortic valve is an operation that was developed to treat patients with aortic root aneurysm with or without aortic insufficiency, and it is used, at least in my hands, mostly in patients with Marfan syndrome, Loeys-Dietz syndrome, or forme fruste Marfan, but the average age of our patients is almost half of yours.

A supra-annular repair of aortic root aneurysm such as the operation you described works very well, just like remodeling works very well; actually, you are doing a modified remodeling, but instead of tailoring a sinus in the end of a graft, you are tailoring the sinuses individually.

I do not think annular dilatation developed in any of your patients. First, your follow-up is too short. It would take much longer than an average of 43 months to see annular dilatation by echocardiography. I have been unable to detect predictors of annular dilatation in young patients in my practice who had the remodeling procedure. We have not seen dilation of the aortic annulus in older patients in whom remodeling such as what you described was done.

I believe that the main reason your results are so good is that you are selecting your patients carefully. In other words, they are patients who have a normal aortic annulus and they are older. It is widely known that the aortic root dilation is usually asymmetrical, and the noncoronary sinus is often the first one to dilate. Actually, in patients with aortic root aneurysms owing to a bicuspid aortic valve, almost invariably the posterior sinus is the first one to dilate. Therefore, if you do not have annular dilatation to start with, whatever technique is used should work well. I believe that remodeling such as you described or remodeling the way Sir Magdi Yacoub described would provide equally good results if the annulus does not dilate.

In your series, only 9 patients had Marfan syndrome. How many patients in your series were younger than 30 years of age, and were they operated on because of degenerative aortic root aneurysm or other disease?

Dr Urbanski. Thank you for the kind comments, Tirone. I completely agree with you that the mean age of our population is a little bit higher than in other reports. Since we do not have a pediatric department in our cardiac center, we do not see many of these young patients. The portion of those who are younger than 40 years is only $10 \%$.

I also agree with you that the number of Marfan patients was very low in our population. However, I do not believe that the share of Marfan patients is a good mark for efficacy of reconstructive aortic surgery, because there is enormous diversity of disease in Marfan patients. Some such patients have changes of all 3 cusps with very low positioned commissures. In my opinion, they are not suitable for repair at all. On the other hand, there are patients such as the patient I demonstrated in my third slide. He was a Marfan patient without aortic regurgitation but with isolated dilation of the aortic root to about $5 \mathrm{~cm}$ and an annulus size of 3 . I operated on him because he had severe mitral regurgitation. I also remodeled the root, surely with all 3 sinuses. I do not think that any downsizing of this aortic root would have been a good solution because an overcorrection of the valve with a very good coaptation height of about $1 \mathrm{~cm}$ could lead to shrinkage of the cusps. I think that in such cases remodeling is a good solution, because without surgery the natural progression would lead to irregular dilatation of the 
sinuses in all directions and to secondary changes of the cusps, with tension along the free margin and with secondary fenestrations or the development of fibrous folds. A repair of sinuses in such patients prevents the progression and the occurrence of events.

However, what we meet in our everyday surgical life are not Marfan patients. Instead, we see patients about 50 or 60 years old with degenerative disease, with hypertension, with more or less pronounced cusp disease. This is the disease that I aim to address with the particular technique I developed. The effect of this technique is that I repaired 100 aortic valves last year and expect 150 this year.

Dr David. That is wonderful. Unfortunately, the title of your paper is misleading, that a supraannular repair solves all problems of the patient with aortic root aneurysm. It does not, particularly in young patients in whom the annulus can dilate, and the bicuspid aortic valve as well. Incompetent bicuspid aortic valve, by and large, is associated with annular dilation.

You had to repair the aortic cusps in 106 patients, or 45\% of them, which is similar to our results. You augmented the height, or changed the cusps, in 68 instances and reduced the annulus with subcommissural plication in 15. Subcommissural plication is an annuloplasty. You reinforced the free margin in 12 and plicated the central portion in 25 , a total of 128 repaired cusps. So more than half of your patients had repaired cusps, and repaired cusp was a predictor of a bad outcome. It has not been in my hands, it has not been in other hands, and I bet the difference is that we repair cusps concomitant with the reimplantation technique. If you have to replace 1 cusp with pericardium in a 62-year-old patient, isn't it better to replace the whole valve with a pig valve? Newer pig valves last 15 to 20 years in most 63-year-old patients.
Dr Urbanski. Actually, what I do is not augmentation. I normalize the previous shape and size of the cusp, because I use the natural frame of the cusp consisting of free margin, the commissures, and the annulus. I am completely aware that durability of autologous pericardium is limited, but the same is true for the native cusps. If the cusps are changed, shrunk, and have fibrous folds or other pathologic conditions and if you leave such cusps in place, you can also expect recurrent regurgitation. Gebrine (Dr El Khoury) demonstrated 1 year ago a population with the complex aortic valve and root reconstruction, and in his group only $85 \%$ of patients had freedom from aortic regurgitation of greater than $2+$ at 5 years. I think it is well comparable with our results.

In general, I do not expect a lifelong solution for such valve repairs, but I believe we are offering a solution for maybe 10 to 15 years.

Dr David. That is my point. Is this procedure better than a tissue valve? Surgeons who do not do 150 aortic valve repairs per year may serve their patients better by replacing the valve with a porcine bioprosthesis.

Finally, more a comment than a question: With a mean followup of only 3.5 years, one has to be a bit more cautious about interpretation of the results. When I introduced aortic valve-sparing operations, I thought that we could use both reimplantation and remodeling without differences in outcomes. It took us 10 to 12 years of follow-up to see dilatation of the root after remodeling of the aortic root. Dilation of the annulus does not occur overnight in a bicuspid aortic valve or in patients with aortic root aneurysm. Therefore, 3.5 years is really intermediate results.

Dr Urbanski. I agree completely. I will follow my patients and come back in 5 years to report again to this Association. Thank you very much. 


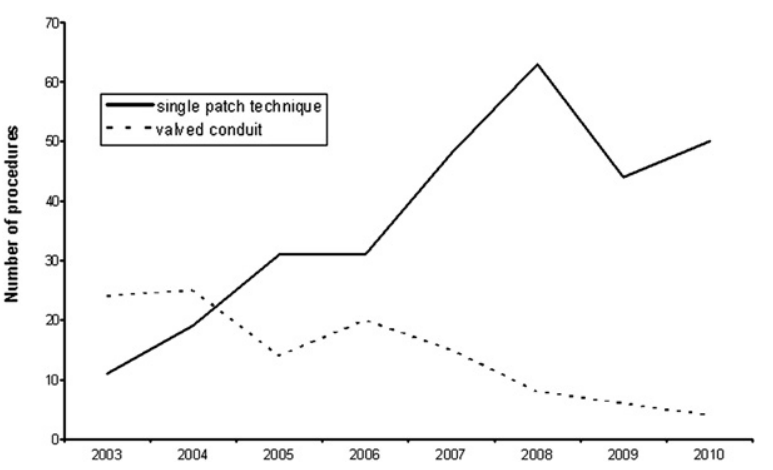

FIGURE E1. Graphic showing rates of aortic root repair using the singlepatch technique (continuous line) and complete aortic root replacements with valved conduits (dotted line). For details, see text. 